\title{
U tilidad de la terapia trombolítica con estreptokinasa a dosis baja en infarto agudo del miocardio
}

\author{
Carlos Raffo $\mathrm{G}^{1}$, Jorge Bartolucci J ${ }^{1}$, Ramón Corbalán $\mathrm{H}^{2}$, \\ Mauricio Aninat $\mathrm{H}^{1}, \mathrm{H}$ umberto Torres $\mathrm{H}^{1}$, Juan $\mathrm{C}$ arlos \\ Prieto $D^{3}$, Fernando Lanas $Z^{4}$, Eduardo Chávez $\mathrm{S}^{5}$, \\ Francisco C umsille $\mathrm{G}^{\mathrm{a}}$, Carolina $\mathrm{Nazzal} \mathrm{N}^{\mathrm{b}}$, \\ y G rupo de Estudio Multicéntrico del Infarto (G EMI)*
}

\section{U sefulness of thrombolytic therapy with low doses of streptokinase in acute myocardial infarction}

Background: The optimal dose of Streptokinase in the treatment of acute myocardial infarction is not well established. Apparently, the thrombolytic efficacy would not increase with doses over 750.000 units. Aim: To compare the effectiveness and safety of treatment with low doses of Streptokinase, ranging from 500.000 to 750.000 units, in patients with ST elevation acute myocardial infarction. Patients and methods: From September 1993 to September 1998, the GEMI register of patients with acute myocardial infarction, was carried out in 37 hospitals, incorporating 4,938 patients. Of these, 1,631 patients received streptokinase. According to the administered dose of Streptokinase, patients were divided in two groups: 1,465 patients who received 1.5 millions $U$ in 60 minutes (classical therapy group), and 166 patients with ischemic chest discomfort and either ST-segment elevation or left bundle-branch block on the electrocardiogram, who received 500.000 to $750.000 \mathrm{U}$ streptokinase administered in no more than 30 minutes, with heparin, within 0 to 6 hours of symptom onset. Successful reperfusion, mortality, complications, and hospital outcome was evaluated in both groups. Results: The low dose group of patients had a better reperfusion criteria profile. No differences between groups were observed in patient evolution, mortality, maximum Killip classification, post myocardial infarction heart failure, ischemic complications, arrhythmias or mechanical complications. Conclusions: These results suggest that streptokinase in low doses is at least as effective as classical therapy, in the treatment of ST elevation acute myocardial infarction (Rev Méd Chile 2006; 134: 1249-57).

(Key words: Myocardial infarction; Streptokinase; Thrombolytic therapy)

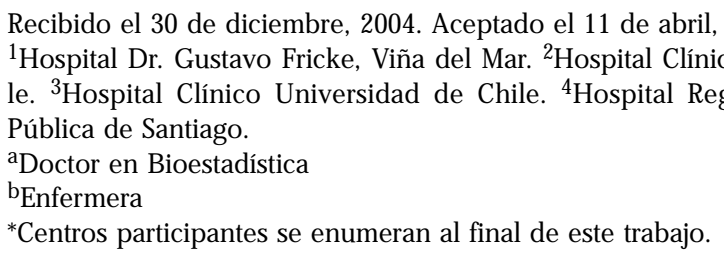

Correspondencia a: Dr. Jorge Bartolucci J. Jefatura de Cardiología y Cirugía Cardiovascular, Clínica Santa María. Avenida Santa María 0500 3o piso, Santiago de Chile. E mail: jbartolucci@csm.cl

Dr. Carlos Raffo. Departamento de Vínculos, Sección de Cardiología,

Servicio de Medicina, Hospital Clínico Dr. Gustavo Fricke. Álvarez

1532, Viña del Mar, Chile. E mail: docraffo@yahoo.com. 
$\mathrm{L}$ a terapia trombolítica ha tenido gran impacto en el manejo del infarto agudo del miocardio (IAM), en especial al reducir la mortalidad ${ }^{1}$, con una reducción de $20 \%$ a $25 \%$ del riesgo relativo a los 30 días, equivalente a 7-8\% del riesgo absoluto en los diversos estudios clínicos ${ }^{2}$. En la actualidad, el grado de reperfusión (TIMI 2-3) de la arteria afectada y la precocidad ${ }^{3}$ con la cual se logra la reperfusión, guardan una estrecha relación con la sobrevida de los pacientes, calculando que se requieren 20 arterias abiertas para salvar una vida 4 , a este respecto los activadores del plasminógeno tisular (t-PA), en sus diversas formas, han superado a la estreptokinasa (SK) ${ }^{2}$, excepto cuando ésta se utiliza en asociación con heparina, pero el riesgo de hemorragia intracraneana (HIC) hace prohibitivo su uso ${ }^{5}$. Uno de los grandes problemas del empleo de los t-PA en los países menos desarrollados es su alto costo, situación que impide su uso frecuente en gran parte de Sudamérica, incluso, la terapia con SK no está disponible en muchos de los centros hospitalarios de nuestro país. La SK se utilizó inicialmente por vía intracoronaria en dosis baja (200.000 a $250.000 \mathrm{U}$ ) y, posteriormente, por vía endovenosa sistémica en dosis mayores; el concepto de la terapia a eltas dosis y breve duración»se inició con 500.000 a 1.000 .000 U de SK en 30 a $60 \mathrm{~min}^{6}$. La dosis óptima de SK endovenosa no ha sido hasta ahora bien definida, pero los trabajos más relevantes han empleado dosis de 1.500.000 U ${ }^{7-9}$. La dosificación de la SK es compleja, dado que, por ser un activador indirecto del sistema fibrinolítico, requiere de una adecuada relación entre la SK unida a plasminógeno y el plasminógeno libre, susceptible de ser activado a plasmina ${ }^{10}$. Aparentemente, no se logranía un incremento de la eficacia trombolítica con dosis superiores a 750.000 $\mathrm{U}^{11}$. En un estudio clínico previo, en un grupo pequeño de pacientes, utilizando dosis de $500.000 \mathrm{U}$ de SK, se demostró actividad fibrinolítica intensa en $60 \%$ de los casos ${ }^{12}$.

En algunos centros hospitalarios de nuestro país, en un intento de disminuir los costos de esta terapia, se ha realizado un protocolo basado en la utilización de SK en dosis de 500.000 a 750.000 U, administrada en 30 min y asociada a heparina de bajo peso molecular (HBPM). La asociación de HBPM a la terapia se basa en la evidencia de un aumento paradójico en la actividad de la trombina luego del uso de $\mathrm{SK}^{13}$, existiendo tendencia a la trombosis y a la reconstitución del coágulo coronario ${ }^{14}$, por tanto el empleo de heparina podría contribuir al éxito de la terapia trombolítica, debido a su efecto inhibidor de la trombina ${ }^{14}$.

Objetivo: comparar eficacia y seguridad del tratamiento trombolítico con estreptokinasa en baja dosis, equivalente a $500.000 \mathrm{U}-750.000 \mathrm{U}$, con la terapia clásica de 1.500 .000 U en IAM con supradesnivel del segmento ST.

\section{MATERIAL Y MÉTODO}

En agosto de 1993 se puso en marcha en Chile el registro nacional del infarto agudo del miocardio, conocido como GEMI (Grupo de Estudio Multicéntrico del Infarto Miocárdico), participando en él 37 hospitales de todo el país, los que realizaron el registro y estudio prospectivo de los pacientes con IAM, de acuerdo a una ficha tipo, descrita en publicaciones anteriores.

El período de registro fue entre agosto de 1993 y diciembre de 1995, incorporando 2.957 pacientes; se efectuó un segundo período de registro durante los años 1997 y 1998, ingresando 1.981 pacientes. De la totalidad de estos pacientes, 1.631 recibieron terapia trombolítica con SK. Fueron incluidos los pacientes que ingresaron con el diagnóstico de IAM y que cumplían con, al menos, 2 de los siguientes criterios: a) dolor retroesternal prolongado (duración $>30 \mathrm{~min}$ ); b) elevación del segmento ST en 2 o más derivaciones contiguas del ECG; c) aumento característico de las enzimas creatinfosfoquinasas (CK) y de su fracción $\mathrm{MB}$ (CK-MB).

Todos los pacientes fueron trombolizados durante las primeras $6 \mathrm{~h}$ desde el inicio de los síntomas, debiendo cumplir los criterios clásicos para la trombolisis y no presentar contraindicaciones. Igualmente, todos recibieron una dosis oral, a masticar, de ácido acetilsalicílico (AAS) de 200 miligramos, previo al inicio de la terapia trombolítica. El grupo de terapia clásica o estreptokinasa a dosis estándar (SKDS) recibió 1,5 millones U en un período de 60 min y el grupo de baja dosis (SKBD) se trombolizó con 500.000 a 750.000 U de SK, a pasar en un período no superior a $30 \mathrm{~min}$. En el grupo de SKBD se asoció a la trombolisis, en los 
primeros pacientes trombolizados (30\%), el uso de heparina convencional a dosis plena, en ellos se llevó el PTTK a un valor equivalente al doble del valor normal, en los restantes pacientes se utilizó HBPM (Fragmin ${ }^{\circledR} 5.000 \mathrm{U}$ cada $12 \mathrm{~h}$ o Fraxiparine ${ }^{\circledR}$ 0,6 MG cada $12 \mathrm{~h}$ ). La utilización de heparina clásica o HBPM se continuó hasta las $72 \mathrm{~h}$ postrombolisis. Ambos grupos recibieron, además, terapia médica antianginosa y de remodelación, de acuerdo con las indicaciones de sus médicos tratantes. Las características clínicas con respecto a edad, sexo, factores de riesgo cardiovascular, localización del infarto y estado funcional según clasificación de Killip previo a la trombolisis de ambos grupos, se registraron de acuerdo con el protocolo previamente expuesto ${ }^{15}$.

Se consideraron como eventos cardiovasculares el desarrollo de Killip igual o mayor a III o insuficiencia cardíaca, arritmias supraventriculares (fibrilación auricular o flutter y enfermedad del nódulo sinusal), arritmias ventriculares (taquicardia ventricular, fibrilación ventricular), alteraciones de la conducción auriculoventricular (bloqueo auriculoventricular de $2^{\circ}$ o $3^{\mathrm{er}}$ grado y desarrollo de nuevo bloqueo completo de rama izquierda), complicaciones mecánicas (ruptura ventricular, insuficiencia mitral, comunicación interventricular) y muerte de origen cardíaco.

La reperfusión clínica se evaluó basándose en 3 parámetros y 4 puntos, como previamente hemos descrito 16 :

1. Parámetro dolor: regresión del dolor de al menos $50 \%$ durante los primeros $90 \mathrm{~min}$, en una escala del 1 al 10.

2. Parámetro electrocardiograma (EKG): regresión del supradesnivel de ST de al menos 50\%, durante los primeros $90 \mathrm{~min}$, o aparición de onda T negativa en las primeras $24 \mathrm{~h}$.

3. Parámetro enzimas: alza enzimática máxima de $\mathrm{CK}-\mathrm{Mb}$ durante las primeras $12 \mathrm{~h}$.

Se tipificaron como eventos adversos de la trombolisis el desarrollo de accidente cerebrovascular (AVE), hipotensión y reacciones alérgicas.

Se definieron como puntos finales muerte y reinfarto, dado a que éstos no se prestan a diagnósticos equívocos o dependientes del observador.
Estadística. Se utilizó la prueba de Chi cuadrado para el estudio de las diferencias de las características demográficas y clínicas, así como también para el análisis de los criterios de reperfusión y evolución intrahospitalaria de ambas dosis de SK. Para la comparación de los promedios de edad en ambos grupos, se utilizó la prueba $t$ de Student. Un valor de $\mathrm{p}<0,05$ fue considerado significativo.

El análisis de los datos se realizó mediante el programa estadístico SAS (Statistical Analysis System).

\section{RESULTADOS}

El grupo de terapia clásica SKDS (G1) correspondió a 1.465 pacientes y el grupo de SKBD (G2) a 166 pacientes. Las características clínicas con respecto a edad, sexo, factores de riesgo cardiovascular, localización del infarto y Killip previo a la trombolisis de ambos grupos, se muestran en la Tabla 1. En ella podemos observar que ambos grupos previos a la trombolisis son bastante comparables, no existiendo diferencias estadísticamente significativas en los datos estudiados, salvo un mayor porcentaje de HTA (Figura 2), en el grupo de pacientes de SKBD (G1: 40,14 v/s G2: 50,6 p <0,01) y de obesidad en los pacientes de SKDS (G1: 22,59 vs. G2: $11,45 \mathrm{p}<0,01$ ). Al considerar el grupo de mayor riesgo post-IAM, que corresponde a los pacientes mayores, entre 65 y 75 años, observamos también que ambos grupos son semejantes (Figura 1).

Al analizar los criterios de reperfusión (Tabla 2) queda en evidencia que existió un mayor porcentaje de criterios aislados a favor del grupo de baja dosis, con excepción del alza precoz de CK-MB, donde fueron semejantes. Al analizar el número de criterios presentados en cada paciente, sólo para la suma de dos criterios fue superior el porcentaje en el G1 ( 28,25 vs 17,52 , p <0,01), en todas las otras combinaciones el porcentaje fue mayor en el G2, pero sin alcanzar significación estadística.

$\mathrm{Al}$ agnuparlos con 1 ó 2 criterios y 3 ó 4 criterios de reperfusión, se evidencia que el grupo G2 presentó un franco predominio de tres o más criterios 
Tabla 1. Características clínicas iniciales de los pacientes sometidos a trombolisis con SK ( $\mathrm{n}=1.631)$

\begin{tabular}{|lccc|}
\hline & $\begin{array}{c}\text { Grupo } 1 \\
(\mathrm{SK}=1,5 \text { millones } \mathrm{U}) \\
\mathrm{n}=1.465\end{array}$ & $\begin{array}{c}\text { Grupo } 2 \\
(\mathrm{SK}=500 \text { a } 750 \text { mil U) } \\
\mathrm{n}=166\end{array}$ & $\mathrm{p}$ \\
\hline Factores poblacionales & & & \\
Edad (media + DS) & $58,56+12,09$ & $58,96+11,42$ & NS \\
Mayores de 65 años (\%) & 28,64 & 29,52 & NS \\
Mayores de 75 años (\%) & 8,14 & 9,04 & NS \\
Sexo (M/F) & $80,52 / 19,48$ & $74,7 / 25,3$ & \\
Factores de riesgo CV (\%) & & & $<0,01$ \\
Hipertensión arterial & 40,14 & 50,6 & NS \\
Diabetes Mellitus & 16,79 & 15,66 & NS \\
Dislipidemia & 23,41 & 21,69 & NS \\
Tabaquismo & 51,31 & 55,76 & $<0,01$ \\
Historia familiar & 15,43 & 21,08 & \\
Obesidad & 22,59 & 11,45 & NS \\
Antecedentes CV previos (\%) & & & NS \\
IM previo & 8,91 & 10,3 & NS \\
Angor pectoris & 40,18 & 50 & NS \\
ACTP & 0,92 & 0,6 & NS \\
BAC & 1,4 & 1,2 & \\
Insuficiencia cardíaca & 3,04 & 2,45 & NS \\
Características al ingreso (\%) & & & NS \\
IAM anterior & 51,44 & 47,27 & NS \\
IAM inferior & 47,43 & 52,73 & NS \\
Killip I a II & 93,52 & 95,18 & 4,82 \\
Killip III a IV & 6,48 & & \\
\hline
\end{tabular}

M/F: Masculino/Femenino. CV: Cardiovasculares. IM: Infarto miocárdico. IAM: Infarto agudo del miocardio. ACTP: Angioplastia coronaria transluminal percutánea. BAC: By-pass aorto-coronario.

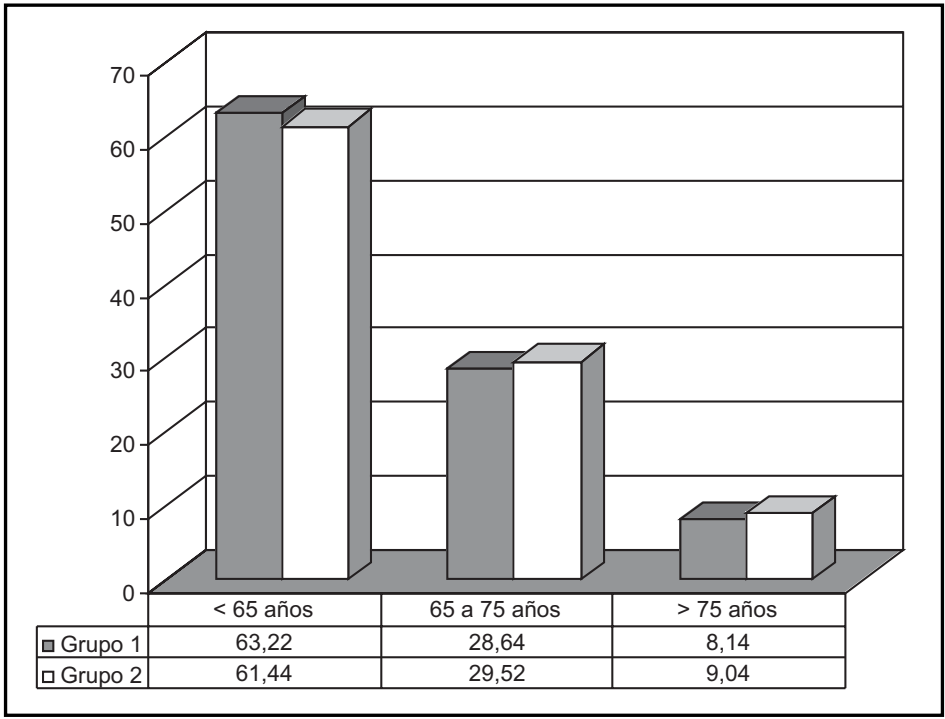

FIgURA 1. Distribución porcentual por edad. 
de reperfusión (73,13\% v/s: 51,01\%, p $\varangle 0,0001)$. El nivel de corte de 3 o más criterios se correlacionó con un mejor resultado de reperfusión, como previamente nuestro grupo lo ha demostrado ${ }^{16}$.

No se observaron diferencias estadísticamente significativas para muerte y re-infarto, eventos que no están sujetos a la subjetividad de la interpretación clínica, a diferencia de lo que podría ocurrir con el angor posinfarto. Aunque existió una leve tendencia a menor mortalidad en el grupo dos (G1: 11,26 v/s G2: 9,64), ésta no fue estadísticamente significativa. Del mismo modo

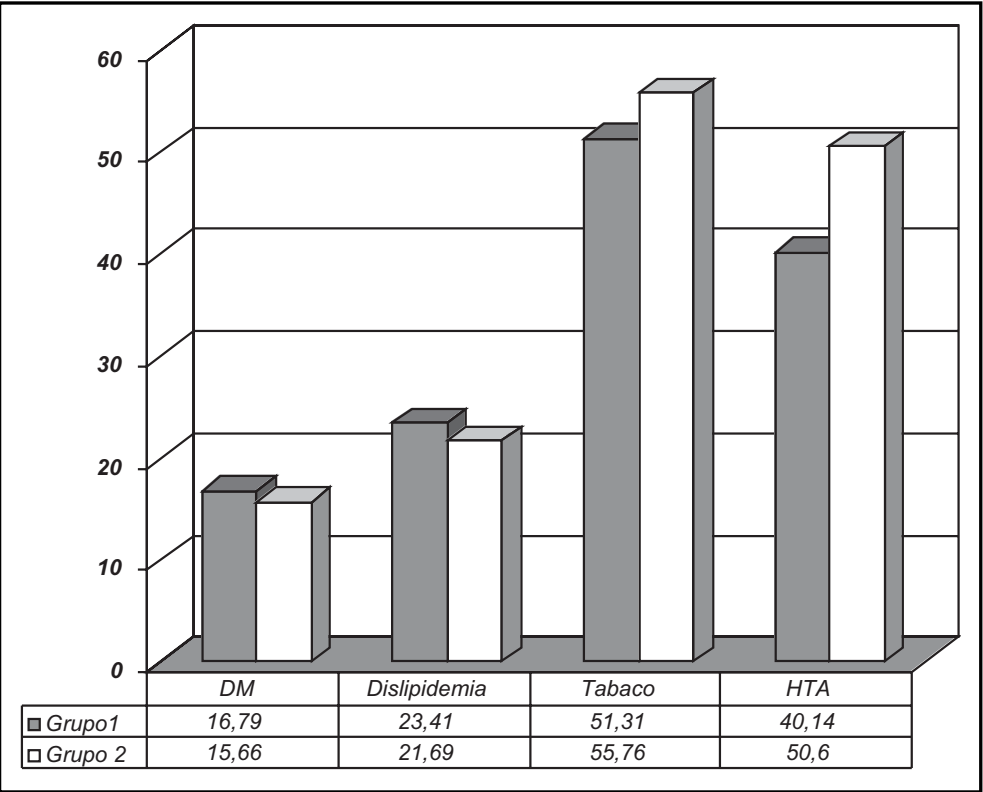

FiguRa 2. Factores de riesgo cardiovascular.

Tabla 2. C riterios de reperfusión de ambos grupos

\begin{tabular}{|c|c|c|c|c|}
\hline & & $\begin{array}{c}\text { Grupo } 1 \\
(\mathrm{SK}=1,5 \text { millones } \mathrm{U})\end{array}$ & $\begin{array}{c}\text { Grupo } 2 \\
(\mathrm{SK}=500 \text { a } 750 \text { mil } \mathrm{U})\end{array}$ & $\mathrm{p}$ \\
\hline \multirow[t]{5}{*}{ A) } & Criterios aislados & & & \\
\hline & Disminución del dolor & 59,11 & 71,6 & $<0,01$ \\
\hline & Disminución del ST & 50,75 & 71,6 & $<0,01$ \\
\hline & Onda T (-) precoz & 46,84 & 59,88 & $<0,01$ \\
\hline & Peak CK-Mb precoz & 59,86 & 57,86 & NS \\
\hline \multirow[t]{5}{*}{ B) } & Criterios conjuntos & & & \\
\hline & 1 criterio & 17,78 & 8,74 & NS \\
\hline & 2 criterios & 28,25 & 17,52 & $<0,01$ \\
\hline & 3 criterios & 29,42 & 37,96 & NS \\
\hline & 4 criterios & 24,55 & 35,33 & NS \\
\hline \multirow[t]{3}{*}{ C) } & Criterios agrupados & & & \\
\hline & $\leq 2$ criterios & 48,99 & 26,87 & $<0,0001$ \\
\hline & $\geq 3$ criterios & 51,01 & 73,13 & $<0,0001$ \\
\hline
\end{tabular}


Tabla 3. Evolución intrahospitalaria en ambos grupos

\begin{tabular}{|c|c|c|c|}
\hline & $\begin{array}{c}\text { Grupo } 1 \\
(\mathrm{SK}=1.5 \text { millones } \mathrm{U})\end{array}$ & $\begin{array}{c}\text { Grupo } 2 \\
(\mathrm{SK}=500 \text { a } 750 \text { mil U) }\end{array}$ & $\mathrm{p}$ \\
\hline \multicolumn{4}{|l|}{ Puntos finales } \\
\hline Mortalidad intrahospitalaria & 11,26 & 9,64 & NS \\
\hline Mortalidad Hosp + Re - IAM & 13,49 & 13,28 & NS \\
\hline \multicolumn{4}{|l|}{ Complicaciones isquémicas } \\
\hline Angor posinfarto & 15,33 & 14,55 & NS \\
\hline Re-infarto & 2,23 & 3,64 & NS \\
\hline \multicolumn{4}{|l|}{ Evolución de FVI } \\
\hline Killip máximo I-II & 84,85 & 87,35 & NS \\
\hline Killip máximo III-IV & 15,15 & 12,65 & NS \\
\hline Desarrollo de IC & 19,44 & 12,73 & NS \\
\hline \multicolumn{4}{|l|}{ Complicaciones arrítmicas } \\
\hline Ritmo nodal & 9,3 & 4,58 & NS \\
\hline CPV frecuentes & 34,12 & 7,36 & $<0,01$ \\
\hline TV sostenida & 10,84 & 6,06 & NS \\
\hline \multicolumn{4}{|l|}{ Complicaciones mecánicas } \\
\hline $\mathrm{IM}^{\circ}$ aguda & 1,40 & 1,22 & NS \\
\hline CIV & 0,84 & 1,83 & NS \\
\hline Ruptura VI & 1,05 & 1,83 & NS \\
\hline Total & 3,13 & 4,82 & NS \\
\hline \multicolumn{4}{|l|}{ Otras complicaciones } \\
\hline AVE & 8,66 & 8,97 & NS \\
\hline Hemorragia mayor $\left(\mathrm{N}^{\circ}\right)$ & 3 & 0 & NS \\
\hline Hipotensión $\left(\mathrm{N}^{\circ}\right)$ & 49 & 7 & 0,00006 \\
\hline
\end{tabular}

Mortalidad Hosp: Mortalidad intrahospitalaria. VFI: Función ventricular izquierda. IC: Insuficiencia cardíaca. CVP: Complejos ventriculares prematuros. VFI: Función ventricular izquierda. IC: Insuficiencia cardíaca. CIV: Comunicación inter-ventricular. VI: Ventrículo izquierdo. AVE: Accidente cerebrovascular.

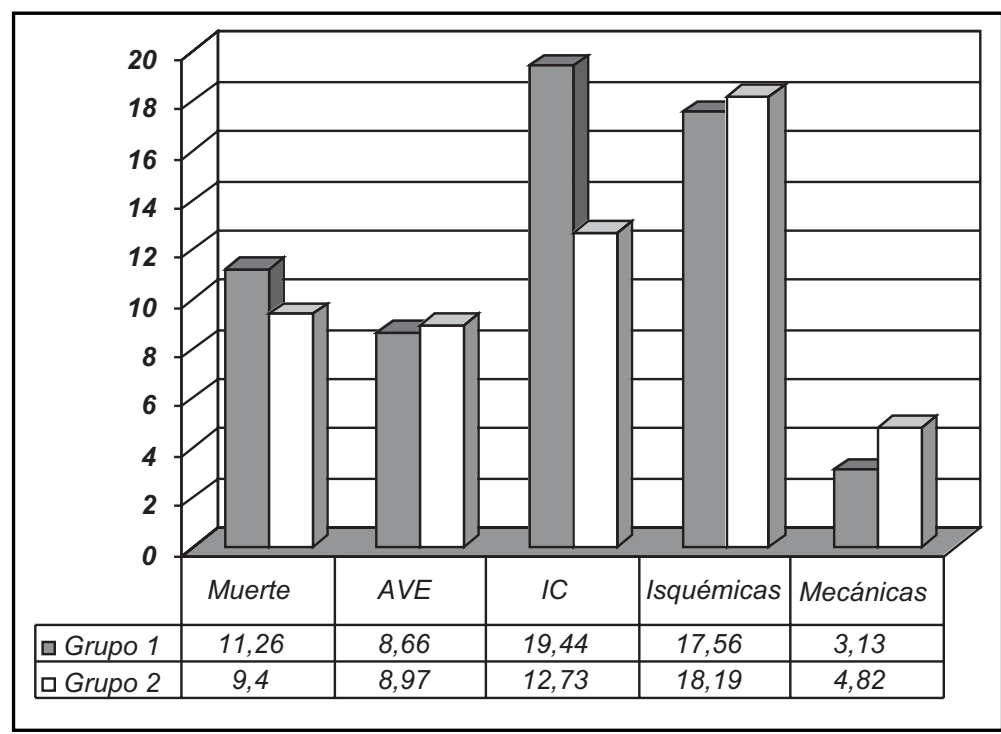

FIGURA 3. Complicaciones postrombolisis. 
no se encontraron entre ambos grupos diferencias respecto a las complicaciones isquémicas, eléctricas o mecánicas (Tabla 3; Figura 3), con la sola excepción de un mayor porcentaje de complejos ventriculares prematuros frecuentes en el G1.

Desde el punto de vista de la evolución de la función ventricular izquierda postrombolisis, no hubo diferencias significativas entre ambos grupos, tanto en el nivel máximo de Killip alcanzado, como en el posterior desarrollo de insuficiencia cardíaca clínica (Tabla 3), aunque un mayor porcentaje de pacientes del G1 presentó insuficiencia cardíaca sin resultar estadísticamente significativa $(19,44 \mathrm{v} / \mathrm{s} 12,73)$

No existieron tampoco diferencias en el número de pacientes con accidente cerebrovascular en ambos grupos, aunque cabría esperar que los pacientes del grupo dos respecto al grupo uno, hubieran tenido mayor riesgo hemorrágico, por tener un mayor porcentaje de pacientes mayores, mayor proporción de hipertensión arterial y la utilización de heparina asociada a la trombolisis.

El número de pacientes con complicaciones hemorrágicas mayores fue muy pequeño como para obtener conclusiones válidas. Igualmente, aunque estadísticamente $(p<0,00006$ ) el grupo dos tiene 7 veces menos hipotensión que el grupo uno, la escasa frecuencia de esta complicación (56 casos) nos hace dudar de su validez, a pesar de ello, la SKBD es aparentemente mejor tolerada que la SKDS, basado en el hecho de que un menor número de pacientes presentaron hipotensión.

\section{Discusión}

Si bien hay una marcada diferencia numérica de pacientes entre ambos grupos (alta y baja dosis), ambos son semejantes respecto a sexo y porcentaje de pacientes con diabetes mellitus (Tabla 1), factores todos que pueden incidir en el pronóstico del IAM. Sin embargo, el grupo dos, correspondiente a SKBD, se encuentra en leve desventaja, por tener un mayor porcentaje de pacientes hipertensos (G1: 40,14 v/s G2: 50,60, p <0,01), un mayor porcentaje de mujeres (G1: 19,48 v/s G2: 25,30 ), aunque no significativo y una proporción discretamente mayor en el número de pacientes mayores (Tabla 1), lo que no es estadísticamente significativo, pero que sumado a los puntos anteriores, le confiere al G2 un relativo mayor riesgo de accidente cerebrovascular, como lo expone Simoons en su trabajo sobre el riesgo de presentar esta complicación ${ }^{17}$, riesgo que se incrementaría por el uso de heparina asociada a la $\mathrm{SK}^{5}$. Este «mayor riesgo teórico» no se observó en la práctica, este hecho nos plantea la duda de si existe un cierto grado de sprotección»del accidente cerebrovascular, con la utilización de dosis menores de SK.

El presente trabajo sugiere que la terapia con baja dosis de SK, en asociación con heparina, es al menos tan efectiva como la terapia clásica, logrando un mejor perfil de reperfusión clínica, expresado por los criterios agrupados de reperfusión (Tabla 3), sin mayores complicaciones y con la consiguiente disminución de los costos. Estos resultados son concordantes con el trabajo de Tatu-Chitoiu, quien en un protocolo prospectivo randomizado con SK 750.000 U ev más enoxeparina en el manejo prehospitalario del IAM v/s SK heparina convencional, demostró una disminución de la mortalidad a 30 días (5,7\% v/s 10\% p $<0,05$ ), igualmente la reperfusión (no angiográfica) fue mejor en el grupo combinado SK-HBPM $(81 \% \mathrm{v} / \mathrm{s} 57 \%)^{18}$. Otro factor que podría influir en los resultados es la velocidad de infusión, pues es conocido que la infusión rápida favorece la reperfusión, logrando porcentajes de reperfusión de $77 \%$ a $80 \%$ v/s $57 \%$ a $58 \%$ en el esquema clásico 18,19 , si bien estos resultados no han sido confirmados por otros autores ${ }^{20}$. En nuestros pacientes trombolizados con SKBD, ésta se infundió en menos de 30 min e inclusive, en la mayoría, en menos de 15 a 20 min, este hecho podría tener relación con la efectividad de la terapia empleada, que corresponde sólo a un tercio o la mitad de la dosis habituales. Esto nos explicaría, por otra parte, el mayor porcentaje de pacientes con más de 2 criterios de reperfusión en el G2 con respecto al G1. De igual forma, la utilización de HBPM en $70 \%$ de nuestros pacientes podría ser un factor que contribuyó al adecuado resultado de la terapia, lo que es concordante con los trabajos de Tatu-Chitoiu con respecto a la asociación de ambas terapias en la fase aguda 
poststreptokinasa, además de la efectividad de las HBPM en otras formas de presentación del episodio coronario agudo 22,23 , como su reconocida asociación en la terapia de fase tardía del IAM, al no aumentar la tasa de hemorragia mayor ${ }^{24}$. Con respecto a nuestro grupo, esta fue utilizada por más de cinco días postrombolisis.

\section{REFERENCIAS}

1. The Gusto Investigators. An international trial comparing four thrombolytic strategies for acute myocardial infarction. N Engl J Med 1993; 329: 673-82.

2. Ross AM. New plasminogen activators. A clinical review. Clin Cardiol 1999; 22: 165-71.

3. Ross AM, Coyne K, Moreya E, Reiner JS, Greenhouse SW, Walker PL, Somoons ML, Draoui YC, Califf RM, Topol EJ, Van De Werf F, Lundergan CF, for the Gusto-I Angiographic Investigators. Extended mortality benefit of early postinfarction reperfusion. Circulation 1998; 97: 1549-56.

4. Simes RJ, Topol EJ, Holmes DR, White HD, Rutsch WR, Vahanian A, Simoons ML, Morris D, Betriu A, CALIFF RM, Ross AM, for the GUSTO-I Investigators. Link between the angiographic substudy and mortality outcomes in the large randomized trial of myocardial reperfusion. Circulation 1995; 91: 1923-8.

5. Antman EM, Giuglano RP, McCabe $\mathrm{CH}$, Gibson $\mathrm{M}$, Adgey AJJ, Ghali M, Coussement P, Anderson KM, Scherer J, Van De Werf F, Braunwald E, for the TIMI 14 investigators. Abciximab (reoPro) potentiates thrombolysis in ST elevation myocardial infarction: Results of TIMI 14 trial. J Am Coll Cardiol 1998; 31(suppl): 191A.

6. Schroeder B, Von Leitnere, Linder T. Intravenous short-time thrombolysis in acute myocardial infarction (Abstract). Circulation 1981; 64: (suppl IV) 10.

7. GISSI (Gruppo Italiano por lo Studio della Streptochinasi nell'Infarto Miocardio). Effectiveness of intravenous thrombolytic treatment in acute myocardial infarction. Lancet 1986; I: 397-401.

8. ISIS-2. Collaborative Group (Second International Study of Infarct Survival). Randomized trial of intravenous streptokinase, oral aspirin, both or neither among 17.189 cases of suspected acute myocardial infarction: ISIS-2. Lancet 1988; 2: 349-60.
Podemos concluir de nuestro estudio, que la terapia con estreptokinasa a dosis baja, asociada a heparina de bajo peso molecular, es al menos tan efectiva como la terapia clásica y aparentemente mejor tolerada, produciendo adicionalmente un fuerte impacto en la reducción de los costos de la terapia trombolítica.

9. ISAM Study Group. A prospective trial of intravenous streptokinase in acute myocardial infarction (ISAM): mortality, morbidity and infarct size at 21 days. N Engl J Med 1986; 314: 1465-71.

10. Gaffney P, Joe F, R0we E, Whitaker A. The influence of various combination of plasminogen and streptokinase on fibrinolysis. Rate Study. Haemostasis 1981; 10: 304-14.

11. SpanN J, SherRy S. Coronary thrombolysis for evolving myocardial infarction. Drugs 1984; 28: 465-83.

12. Aninat M, Col J, Rankin J, Jacouet L, Anwar A, ETIENNE J. Infarto agudo del miocardio: Eficacia trombolítica de $500.000 \mathrm{U}$ de estreptokinasa asociada a heparina. Rev Méd Chile 1990; 118: 862-67.

13. EISENBERg P, Sherman L, JafFe A. Paradoxical elevation of fibrinopeptide A after streptokinase: Evidence for continued thrombosis despite intense fibrinolysis. J Am Coll Cardiol 1987; 10: 527-9.

14. Lew A, Cercet B, Hord H, Shah P, Ganz W. Usefulness of residual plasma fibrinogen after intravenous streptokinase for predicting delay or failure of reperfusion in acute myocardial infarction. Am J Cardiol 1986; 58: 680-5.

15. Grupo de Estudios Multicéntricos del Infarto al Miocardio (GEMI). ¿Es posible para la cardiología chilena realizar estudios multicéntricos? Revista Chilena de Cardiología 1993: 12: 15360.

16. Corbalán R, Prieto J, Chávez E, Nazzal C, Cumsime F, KRUCOFF M. Bedside markers of coronary artery patency and short-term prognosis of patients with acute myocardial infarction and thrombolysis. Am Heart J 1999; 138: 533-9.

17. Simoons ML, Maggioni AP, KnatTerud G, Leimberger JD, De Jaegere P, Van Domburg R, Boersma E et als. Individual risk assessment for intracranial hemorhage during thrombolytic therapy. Lancet 1993; 342: 1523-8. 
18. Tatu-Chitoiu G, Guran M, Tatu-Chitolu A, Oprisan M, Cismara O, Firiar T et al. Bolus administration of 750.00 IU SK in association with enoxoparin in the prehospital management of acute myocardial infarction. Paper presented at $1^{\text {st }}$ International Congress on Heart Diseases. New Trends in Research, Diagnosis and Treatment. May 18, 1999, Washington, DC. Vol 75 (11) Nov 2000: 1185-92.

19. Tatu-Chitoiu G, Teodorescu C, Fluerasu A, CäpraRU P, GuRAn M, Dan M et al. Accelerated streptokinase a new thrombolytic regimen in acute myocardial infarction. Rom J Intern Med 1998; 36: 183-96.

20. Tatu-Chitoiu G, Orha I, Teodorescu C, Fluerasu A, Serban L, Bumbu A. Rapid infusion of streptokinase standard dose in acute myocardial infarction is followed by a higher rate of coronary reperfusion than standard protocol. Rom J Intern Med 1997; 35: 47-54.

21. Srimahachota S, Sangwatanaroj S, Boonyaratavej S, Suitichaiyakul T, Ngarmukos P. Efficacy of rapid infusion of streptokinase in patients with acute myocardial infarction. J Med Assoc Thai 2000; 83: 8-12.

22. Antman EM, McCabe CH, Gurfinkel EP, Turpic AG, Bernink PJ, Salem D, Bayes De luna A et al, for the TIMI 11B Investigators. Enoxaparin prevents death and cardiac ischemic events in unstable angina/non-Q myocardial infarction: results of the Thrombolysis in Myocardial Infarction (TIMI) 11B Trial. Circulation 1999; 100: 1593-601.

23. Antman EM, Cohen M, Radiey R, McCabe C, Rush J, Premmereur J et al. Assessment of the treatment effect of enoxaparin for unstable angina/ non-Q myocardial infarction: the TIMI 11BESSENCE meta-analysis. Circulation 1999; 100: 1602-8.

24. Glick A, Kornowski R, Michowich Y, Koifman B, Roth A, Lamiado S et al. Reduction of reinfarction and angina with use of low-molecular-weigth heparin after streptokinase (and heparin) in acute myocardial infarction. Am J Cardiol 1996; 77: $1145-8$.
Agradecimientos:

Se agradece el esfuerzo y apoyo prestado por la Escuela de Salud Pública de la Universidad de Chile, en el análisis estadístico.

Comité Coordinador GEMI: Bartolucci J, Corbalán R, Chávez E, Lamich R, Lanas F, Prieto JC. Enfermera Coordinadora: EU Carolina Nazzal. Análisis Estadístico: Francisco Cumsille (Doctor en Bioestadística). Investigadores por centro: Hospitales Regionales: Hospital Juan Noé, Arica: Iván Criollo, Mario Gatica. Hospital Regional Iquique: Virginia Araya, Ignacio Auger. Hospital Regional Antofagasta: Juan Cotorras. Clínica Antofagasta: Guillermo lllanes, EU Marcela Carmona. Hospital Regional de Copiapó: Mario Contreras, Walter Evans. Hospital Regional de La Serena: Claudio Bugueño. Hospital Regional de Coquimbo: Carlos Saldías, Hans González. Hospital Naval de Valparaíso: Femando Cárdenas, Marcelo Opazo. Hospital Gustavo Fricke: Jorge Bartolucci, Carlos Raffo. Clínica Reñaca: Rienzi Díaz, Mónica Carvajal. Hospital Quilpué: Minerva Jara. Hospital San Felipe: José Pérez. Clínica Miraflores: Pedro Chadid, Jessica Berríos. Hospital Regional de Rancagua: Leopoldo Manníquez, Héctor Díaz. Hospital Regional de Talca: Enrique Mercadal, Patricio Vildósola. Hospital H. Martín de Chillán: Gonzalo Mańn, Gustavo Yanine, EU América Yáñez. Hospital Angol: Brenda Flores, Patricia Rosales. Hospital Victoria: Margarita Figueroa. Hospital Naval de Talcahuano: Alejandro Dapelo. Hospital Regional de Temuco, Universidad de la Frontera: Fernando Lanas, Benjamín Stockins. Hospital Valdivia: Herminia Riquelme, Eduardo Garcés. Hospital Base Osomo: Sergio Potthoff, Guido Mutizabal, EU Cecilia Rivas. Hospital Puerto Varas: Cristián García. Hospital Regional de Puerto Montt: Luis F. del Campo, Jaime Venegas, Claudio Kenner. Hospital Coyhaique: Mauricio Tapia, Osvaldo Abarca, EU Hortensia Poblete. Hospital Regional de Punta Arenas: Guillermo Araneda, Bogdam Liberon. Hospitales Area Metropolitana: Hospital San Juan de Dios: Eleodoro Espinoza, Carmen Andrade. Hospital Salvador: Alvaro Puelma, Francisco Manzur, EU Elena Carreño. Hospital Dipreca: Milton Alcaíno, EU M Teresa Arrau. Hospital Clínico Universidad de Chile: Jorge Yovanovich, Felipe Ashwel, Juan Carlos Prieto. Asistencia Pública: Eduardo Chávez, Mario Ratkam, Marcela Argandoña. Hospital Barros Luco Trudeau: Ligia Gallardo, Rubén Lamich. Hospital Pontificia Universidad Católica: Pablo Castro, Mónica Acevedo, EU Juanita Rosales, Mariana Baeza. Hospital Fach: Rodrigo Hernández, EU: Elizabeth Cortés. 